\title{
La carte d'assuré: efficace, adéquate et économique?
}

\section{Judith Wagner ${ }^{a}$, \\ Hanspeter Kuhn ${ }^{b}$, \\ Monique Gautheyc}

a Dr. sc. hum., responsable eHealth FMH

b avocat, secrétaire général adjoint de la FMH

c spécialiste $\mathrm{FMH}$, membre du Comité central de la FMH, responsable du domaine eHealth
Correspondance:

Dr Judith Wagner FMH

Elfenstrasse 18

CH-3000 Berne 15

Tél. 0313591111

Fax 0313591112

judith.wagner@fmh.ch
Les médias regardent d'un œil de plus en plus critique la nouvelle carte d'assuré, élaborée par la Confédération et que chaque assuré devrait avoir reçue au $1^{\text {er }}$ janvier 2010. Dès le début, des voix se sont élevées pour dire que la carte d'assuré pourrait être contreproductive pour le développement de la cybersanté (eHealth). Il semblerait que le temps leur ait donné raison. La possibilité d'inscrire les données médicales du patient sur sa carte peut l'induire en erreur en laissant miroiter une pseudo-sécurité. D'autre part, les avantages administratifs de la carte d'assuré, si toutefois ils existent vraiment, ne seront probablement pas perceptibles avant 2012 au plus tôt et seulement pour les assureurs.

\section{Contexte}

Le Parlement a approuvé en automne 2004 l'art. 42a LAMal actuellement en vigueur. Ce dernier prévoit une carte d'assuré, avec le nouveau numéro AVS, à utiliser pour la facturation des prestations. Par ailleurs, le patient peut donner son accord pour y faire inscrire des données (d’urgence) médicales. Le législateur voulait ainsi poser dans la LAMaL les premiers jalons d'une carte sensée devenir la carte d'assuré mais susceptible de permettre une évolution vers une carte de santé. La majorité du Parlement souhaitait faire ainsi d'une pierre deux coups avec des économies de coûts et une amélioration de la qualité. Mais les votes minoritaires pointaient déjà du doigt les questions centrales, en suspens à l'époque, et qui se posent encore aujourd'hui: l'idée de vouloir mettre des données médicales sur une carte à puce est-elle une bonne approche? Les avantages de cette carte en justifient-ils les coûts [1]?

Une chose est sûre: en 2004, le Parlement n'a pas osé opter pour une véritable carte de santé. Celle-ci permettrait d'apporter un soutien ciblé à des processus thérapeutiques sûrs et efficaces, indépendamment du répondant des coûts des traitements. Elle serait donc indépendante de la caisse-maladie, de l'assuranceaccidents, de l'assurance militaire, de l'assurance-invalidité ou d'une assurance complémentaire privée. Une carte de santé se rangerait du côté du corps médical, sans entrer dans le monde des assureurs. Mais en fin de compte, la carte d'assuré, comme elle se présente dans la LAMal, relève d'un malheureux compromis.

\section{Prises de position de la FMH}

L'eHealth doit être utile aux patients et aux médecins, telle est la teneur de ce que la FMH a souligné avec insistance dans ses prises de position: «Une stratégie

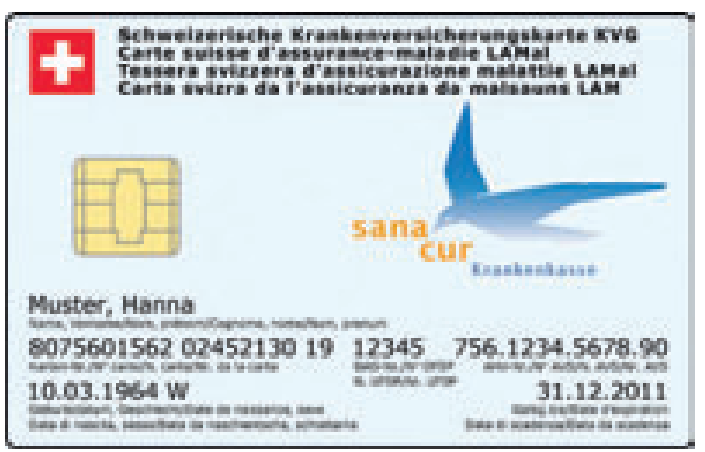

Source: site internet de l'OFSP.

eHealth devrait se développer pour soutenir les processus thérapeutiques et ainsi la relation entre le patient et le médecin. C'est pour nous, un facteur essentiel de réussite de voir dans quelle mesure on arrivera à placer cette relation au centre de la question, à déclarer qu'elle a particulièrement besoin d'être protégée et à soutenir les processus qui l'accompagnent» [2].

Pour diverses raisons, la FMH s'est clairement exprimée contre l'enregistrement isolé de données médicales sur une carte: ce procédé ne permettrait pas de garantir des données actualisées et complètes. Et que se passera-t-il si un patient oublie sa carte, la perd ou si des informations essentielles faisaient défaut? Les données médicales enregistrées sur la carte d'assuré peuvent illusionner le patient avec une dangereuse apparente sécurité.

La FMH a également rejeté le regroupement de la carte d'assuré et de la carte de santé puisqu'il entraîne des inconvénients et des risques considérables. La carte d'assuré, délivrée par l'assureur, reste la propriété de ce dernier. En cas de changement d'assurance, l'assureur peut exiger de l'assuré qu'il lui remette sa carte. Si le patient la perd, toute personne qui l'aurait trouvée pourrait remettre la carte à l'assureur. Il paraît évident qu'aucune donnée médicale n'a sa place sur une telle carte.

Et enfin, la FMH s'est également prononcée contre l'inscription du nouveau numéro AVS sur la carte d'assuré: précisément dans les grandes institutions, les équipes soignantes doivent dès le début noter l'identification administrative du patient pour que l'hôpital puisse ensuite facturer ses prestations. Si elles se servaient du numéro AVS, ce numéro serait en peu de temps connu par «tout le monde». Et pourtant, la révision du système de numéro AVS s'est, en son temps, essentiellement appuyée sur des arguments en faveur de la protection des données. 


\section{Données administratives de la carte}

L'assureur doit inscrire certaines données administratives (cf. fig. 1) sur la carte d'assuré, c'est-à-dire aussi bien en les imprimant sur la carte qu'en les enregistrant sur la puce (le chip).

Par ailleurs, il peut également y enregistrer des données administratives supplémentaires (cf. fig. 1). Les assureurs vont probablement peu mettre des données facultatives sur la carte, notamment parce que beaucoup d'entre elles sont soumises à de fréquentes modifications.

Selon la loi, le fournisseur de prestations doit inscrire le numéro AVS et le numéro d'identification de la carte d'assuré sur la facture [3] lorsque le patient lui présente sa carte [4]. Ces deux numéros peuvent être lus électroniquement sur la puce ou saisis manuellement. Les données administratives enregistrées sur le chip sont accessibles librement, ce qui signifie que l'accès à ces données - à l'inverse des données médicales - ne

\section{Les obligations du médecin}

Lorsque le patient présente sa carte d'assuré, le médecin doit inscrire le numéro AVS et le numéro d'identification de la carte d'assuré sur la facture.

Avant toute procédure de consultation en ligne, le médecin est tenu de demander l'accord du patient. Le procédé en ligne réalisé ne peut avoir lieu qu'au moyen du numéro d'identification de la carte d'assuré et non pas avec le nom, le prénom ou la date de naissance du patient.

Les personnes qui, en leur qualité de médecin, enregistrent des données médicales sur la carte sont tenues d'informer le patient. II doit en particulier savoir quelles personnes sont autorisées à accéder à ses données et à quelles fins, connaître la possibilité de verrouiller ces données au moyen d'un code PIN, ainsi que les avantages et les inconvénients d'un tel verrouillage et être informé de l'intérêt d'effacer ces données avant de restituer sa carte à l'assureur.

\section{Facultatif}

Le patient peut décider s'il souhaite l'enregistrement électronique de ses données médicales sur sa carte. Pour sa part, le médecin peut décider s'il veut proposer cette prestation.

\section{Figure 1}

Données administratives de la carte d'assuré au sens de l'art. 4 al. 1 et 2 OCA.

\section{Données obligatoires}

a. nom et prénom de la personne assurée;

b. numéro d'assuré de l'assurancevieillesse et survivants (AVS);

c. date de naissance de la personne assurée;

d. sexe de la personne assurée

e. nom et numéro d'identification de l'assureur (numéro OFSP);

f. numéro d'identification de la carte d'assuré

g. date d'expiration de la carte d'assuré.

\section{Données facultatives}

a. adresse de la personne assurée

b. adresse de facturation de I'assureur;

c. formes particulières d'assurance au sens de l'art. 62 LAMal;

d. suspension éventuelle de la couverture des accidents en vertu de l'art. 8 LAMal;

e. indications relatives aux assurances complémentaires, pour autant que la personne assurée ait donné son accord;

f. données de la carte européenne d'assurance-maladie. requiert pas l'utilisation d'une carte de professionnel de santé (HPC).

Si ces deux numéros ne figurent pas sur la facture, les assureurs sont en droit de facturer au patient les dépenses administratives supplémentaires occasionnées [5] ils ne peuvent toutefois pas poursuivre le fournisseur de prestations.

La mise en pratique, par contre, risque de se faire attendre: le «Forum Datenaustausch», qui a permis aux partenaires tarifaires de fixer les standards pour l'envoi électronique de factures, a certes trouvé une solution pour intégrer le nouveau numéro AVS et celui de la carte d'assuré dans le formulaire de facturation d'aujourd'hui. Mais plusieurs assureurs ont pourtant signalé qu'ils voulaient attendre la prochaine version de ce formulaire avant de mettre leurs systèmes à jour, c'est-à-dire probablement pas avant 2012. Et pourtant, le législateur n'avait introduit la carte d'assuré que dans le but que les assureurs puissent traiter plus efficacement les factures ...

\section{Données administratives en ligne}

Les assureurs doivent, lors d'une procédure de consultation en ligne, mettre à disposition les indications concernant l'existence d'un contrat d'assurance, la validité de la carte d'assuré ainsi que les données qui doivent figurer sur la carte d'assuré [6]. En plus, l'assureur peut mettre à disposition les données administratives facultatives (cf. figure 1). Les données ne peuvent être interrogées en ligne qu'avec l'accord de l'assuré et la consultation réalisée qu'au moyen du numéro d'identification de la carte d'assuré et non pas, entre autres, avec le nom, le prénom ou la date de naissance du patient.

Les fournisseurs de prestations, par exemple les laboratoires, qui ne voient jamais directement un patient ni sa carte d'assuré pourraient, par le biais de la procédure de consultation en ligne, s'enquérir des données du patient directement auprès de l'assureur. Pour cela, il faudrait par exemple qu'à chaque demande d'examens de laboratoire, l'accord du patient et son numéro de carte d'assuré soient transmis au laboratoire. Sans demandes d'examens de laboratoire électroniques, ou au moins envoyés électroniquement, cela ne semble guère réaliste - et nous en sommes loin aujourd'hui.

Les assureurs mettront, selon toute vraisemblance, les données facultatives uniquement à disposition dans une procédure de consultation en ligne et pas sur la carte elle-même. De plus amples informations à ce sujet suivront ultérieurement.

\section{Données médicales}

Comme le Conseil fédéral tenait à une introduction de la carte d'assuré avec des données médicales au sens de l'art. 42a LAMal, la FMH a essayé d'en influencer le choix et d'obtenir une structure appropriée de ces données - aussi en associant des médecins d'urgence. 
Le «set» de données est calqué sur des données d'urgence mises en place à l'étranger. Des données de types différents peuvent être enregistrées (cf. fig 2). Avec chaque entrée y figurent le numéro GLN (anciennement numéro EAN) de l'auteur de l'inscription et la date de l'enregistrement. Dans le cadre d'une inscription supplémentaire (cf. fig. 2, let. f), il est possible d'indiquer des renseignements sur les dossiers médicaux ou pharmaceutiques à disposition.

Initialement, on avait prévu une multitude d'entrées possibles pour chacun des différents types de données. Mais, en décembre 2009, il s'est avéré que, pour des raisons techniques, il n'y aurait pas de place pour toutes les occurences initialement prévues pour chaque type de données, mais seulement pour un nombre très limité d'entre elles. En l'état actuel de nos connaissances, le nombre maximal d'entrées de données possible pour chacun des différents types de données diffère entre les cartes des divers fabricants.

L'enregistrement électronique de données médicales sur la carte est facultatif pour le patient. Aucun médecin non plus n'est tenu d'inscrire des données médicales sur la carte d'assuré; s'il est disposé à le faire, il peut le facturer dans le cadre de la consultation à titre de prestation obligatoire [7].

Le patient peut verrouiller les données médicales au moyen d'un code PIN, mais rien ne l'y oblige. L'utilisation d'un code PIN assure naturellement une meilleure protection des données. Mais en cas d'urgence, elles pourraient ne pas être accessibles si le patient n'était pas en mesure de saisir son code PIN.

\section{Carte d'assuré et HPC}

Pour accéder aux données personnelles, et plus exactement aux données médicales, le médecin doit être porteur d'une attestation électronique justifiant de sa qualité de fournisseur de prestations sous forme d'une carte de professionnel de santé (HPC). La carte d'assuré et la HPC sont dotées de certificats spéciaux (appelés

\section{Figure 2}

Données médicales («données d'urgence») de la carte d'assuré au sens de l'art. 6 al. 1 OCA et nombre d'entrées possibles en l'état actuel (mise à jour 15.2.2010).

a. données relatives au groupe sanguin et à la transfusion (1);

b. données relatives au système immunitaire (20);

c. données relatives à la transplantation(1);

d. allergies (12);

e. maladies et séquelles d'accidents (20);

f. dans des cas médicalement fondés, inscription supplémentaire $(10+2)$;

g. médication (18);

h. une ou plusieurs adresses de personnes à avertir en cas d'urgence (2);

i. mention de l'existence de directives anticipées (2).
CVC, card verifiable certificates) qui sont reconnaissables d'une carte à l'autre. Depuis la carte d'assuré, une vérification a lieu pour savoir si le CVC figurant sur la HPC est un certificat des groupes de fournisseurs de prestations autorisés. Les directives concernant la procédure d'authentification des cartes et les certificats sont définis dans une ordonnance du Département fédéral de l'intérieur (OCA-DFI). Elle se réfère entre autres au standard technologique eCH0064.

Les certificats nécessaires peuvent être installés ultérieurement sur la carte de professionnel de santé de la FMH (HPC FMH); elle peut faire office de «porteclés» et enregistrer ces certificats spécifiques par le biais d'une fonction internet de téléchargement. Les certificats requis et la fonction de téléchargement ultérieure ont été réalisés dans le cadre du projet de carte de professionnel de santé FMH; l'interopérabilité avec les cartes d'assuré conformes au standard a été testée avec succès.

Actuellement, il y a deux producteurs différents pour la carte d'assuré: Helsana a réalisé une carte d'assuré conforme aux standards en collaboration avec la Poste. Au contraire, SASIS SA, une société qui appartient à santésuisse, et qui produit les cartes pour la plupart des assurances, s'écarte délibérément pour plusieurs points essentiels du standard défini. L'OFSP s'est, pour sa part, contentée d'observer la situation au lieu d'imposer ledit standard. Afin que l'interopérabilité entre les cartes d'assuré non conformes à ce standard et la HPC puisse s'établir, un certificat supplémentaire doit être chargé sur la HPC. Les spécialistes en technologie sont d'avis que cela pourrait fonctionner, même si jusqu'à présent aucune solution de ce genre employant plusieurs certificats ne soit en application sur une carte.

La FMH commandera le développement informatique encore nécessaire à sa carte de professionnel de santé une fois que SASIS et en l'occurence santésuisse auront certifié assumer les coûts supplémentaires qu'ils ont occasionné (leur garantie ne nous est pas encore parvenue au moment de mettre sous presse). Cela signific, que probablement aucune donnée médicale ne pourra être lue ou enregistrée sur les cartes SASIS à court terme.

\section{Questions pratiques ouvertes}

La gestion des données administratives, mais avant tout la gestion des données médicales soulèvent beaucoup de questions pratiques que la FMH a toujours soulignées. Les réponses apportées par l'OFSP ne sont pas convaincantes, elles sont l'expression d'une approche très technique de la problématique, qui néglige de façon impardonnable les processus pratiques. Dans un prochain numéro du BMS, nous consacrerons un article à ce sujet et aux questions de responsabilité juridique.

Aujourd'hui, que ce soit du côté des systèmes informatiques employés au cabinet ou du côté des systèmes de dossiers médicaux électroniques utilisés dans les hôpitaux, aucune application de routine connue ne 
permet de lire et de documenter les données médicales de la carte d'assuré et de les intégrer à un dossier électronique de patient ou inversement de les transférer d'un dossier sur la carte. Seules quelques versions de démonstrations isolées voient le jour ça et là, et elles permettent d'envisager comment cela pourrait fonctionner.

\section{Données médicales de la carte et stratégie eHealth de la Confédération}

A ses débuts, le projet «carte d'assuré» a certainement été porté par des idées visionnaires, mais leur mise en application a ouvert une boîte de Pandore, avec notamment les questions de protection des données, de sécurité et de praticabilité. Et ceci, loin de la réalité quotidienne des cabinets médicaux et des hôpitaux, en raison principalement d'un manque de concertation pour savoir comment ces «instruments» pourraient être intégrés dans les processus administratifs mais aussi thérapeutiques. Cela n'a été en aucun cas un service rendu au eHealth! Seuls les instruments eHealth utiles aux soins du patient arriveront à s'établir. Or, maintenant on produit des charges supplémentaires auprès des fournisseurs de prestations, éventuellement sans même apporter d'avantages administratifs aux assureurs.

La stratégie eHealth de la Confédération prévoit que le médecin habilité par le patient pourra, à l'avenir, accéder à un dossier médical électronique décentralisé. Par contre les données médicales inscrites sur la carte d'assuré ne sont pas une partie importante de la mise en œuvre de la stratégie eHealth. Et comme personne ne semble vouloir faire marche arrière dans ce dossier mandaté par le législateur, la Suisse continuera d'investir des millions dans cette solution, mais est-ce vraiment efficace, adéquat et économique?

\section{Références}

1 Eugen David: (...) «Das zweite Problem, das nicht gelöst ist, ist das Problem der Mutation der Daten. Wenn ich weitere Beispiele nehme: Impfungen oder auch die Adresse des Patienten sind Daten, die permanent ändern können; das gilt auch für Daten bezüglich Krankheiten. Diese Daten müssen dauernd nachgeführt werden. Man muss sich überlegen, ob eine jahresweise Nachführung dieser Daten genügt. Da habe ich Zweifel, wenn man die Karte als Notfallkarte benutzen will. Dann kommt die Frage der Kosten. Wenn Sie solche Karten wegen Mutationen permanent nachführen müssen, entstehen aus dieser ganzen Übung natürlich riesige Kosten. Auch daher habe ich Zweifel, ob dieser Ansatz richtig ist.» (Bulletin E 21.9.2004. p. 459).

Franziska Teuscher: «Aus dem Bericht, den uns der Bundesrat für die Kommissionsdebatte zur Verfügung gestellt hat, geht aber zum anderen klar hervor, dass hinter der Einführung der einheitlichen Versichertenkarte die Absicht steht, in einem zweiten Schritt eine Gesundheitskarte einzuführen. (...) Wir müssen zuerst politisch eine Diskussion darüber führen, was wir mit einer solchen Gesundheitskarte wollen. Denn ich finde es äusserst heikel, wenn wir medizinische Daten auf einer Karte elektronisch speichern.» (Amtl. Bulletin Officiel N 29.9.2004. p. 1503).

2 Position de la FMH au sujet de la stratégie «eHealth», avril 2007.

3 Art. 14 OCA en relation avec l'art. 59 let. d et e OAMal.

4 Art. 10 OCA.

5 Art. 10 OCA.

6 Art. 15 OCA.

7 Cf. les commentaires de l'OFSP concernant l'OCA février 2007: «Les coûts engendrés par l'enregistrement des données visées par l'art. 42a, al. 4, LAMal dans le cadre d'une consultation médicale sont prises en charge par l'AOS.»

\section{Le marché de l'emploi dans le Bulletin des médecins suisses: les postes de médecins en Suisse - mise à jour hebdomadaire.}

Bulletin des médecins suisses Bollettino dei medici svizzeri Schweizerische Ärztezeitun

\section{C'est ici que vous trouverez le poste que vous cherchez ou votre candidat idéal.}

Le Bulletin des médecins suisses, organe officiel de la FMH et de FMH-Services, est un hebdomadaire publiant 45 numéros par an. Il constitue le marché central des offres d'emploi pour les médecins en Suisse.

Toutes les annonces sont publiées immédiatement aussi bien dans la version imprimée que dans la version en ligne sous www.bullmed.ch (en français) et www.saez.ch (en allemand).

Le Bulletin des médecins suisses, revue de pointe des médecins en Suisse, est une publication des Editions médicales suisses $\mathrm{EMH}$.

Nous vous souhaitons le succès! 